\title{
Optimal power allocation in cognitive radio based machine-to-machine network
}

\author{
Haipeng Yao ${ }^{1 *}$, Tao Huang ${ }^{1}$, Chenglin Zhao ${ }^{1}$, Xiaoyong Kang ${ }^{2}$ and Zhongya Liu ${ }^{3}$
}

\begin{abstract}
In this paper, we investigate the optimal power allocation strategy in a cognitive radio-based machine-to-machine (M2M) network where a licensed primary user and multiple unlicensed M2M devices coexist and operate in the same licensed spectrum. We develop a connectivity establishment mechanism in M2M network based on the cognitive radio scheme and propose an optimal power allocation scheme which achieves the maximum system utility while satisfying the QOS requirement of M2M devices and interference constraints of the primary user. The simulation results show that the proposed algorithm achieves better performance.
\end{abstract}

Keywords: Machine to machine; Cognitive radio; Interference constrains; Optimal power allocation; Admission control

\section{Introduction}

It is envisioned that machine-to-machine (M2M) communications are rapidly developing based on the large diversity of machine-type terminals, including sensors, mobile phones, consumer electronics, utility metering, vending machines, and so on. With the dramatic penetration of embedded devices, M2M communications will become a dominant communication paradigm in the communication network, which currently concentrates on machine-to-human or human-to-human information production, exchange, and processing. M2M communications is characterized by low-power, low-cost, and low-human intervention [1].

M2M communications is typically composed of billions of wireless identifiable infrastructure sensors which will be developed and deployed over the coming years. The diversity of the M2M network structures, protocols, and standards, combined with even more diverse application services from users, pose big challenges for M2M network integration and service integration. The capabilities of sensors are generally limited which puts several constraints in M2M communications, including communication spectrum, energy, computation, and storage. These constraints pose a number of unique challenges in the design of network architecture and spectrum

\footnotetext{
* Correspondence: yaohaipeng@bupt.edu.cn

${ }^{1}$ State Key Lab of Networking and Switching Technology, Beijing University of Posts and Telecommunications, Beijing 100876, China

Full list of author information is available at the end of the article
}

usage to achieve a highly connected, efficient, and reliable M2M communication.

The first challenge in M2M communication is the spectrum scarcity. Massive M2M terminals accessing wireless network require lots of spectrum resources, but the exploitable spectrum is becoming scarce resource. Thus, there should be a mechanism to solve the problem of imbalance between the M2M spectrum requirement and the spectrum scarcity.

Another main issue challenges the M2M communication is ever more intensive interference with more radio systems in M2M communication, including unlicensed systems operating in the industrial, scientific, and medical (ISM) frequency band, electronic equipment, and domestic appliances. The performance of M2M communications may be seriously degraded due to the selfexistence/coexistence interference. Moreover, wireless channels in M2M communications are notoriously unreliable due to channel fluctuations and noise, which may become even worse due to the complicated construction in an indoor environment [2].

In this paper, we propose a cognitive radio (CR)-based communication scheme to solve the spectrum scarcity and severe interference problems within $\mathrm{M} 2 \mathrm{M}$ communication. The CR is original from the fact that most of the licensed frequency bands are severely underutilized across time and space in the sense that each licensee is granted an exclusive license to operate in a certain frequency band. The CR, which was first proposed by 
Mitola and Maguire [3], is a promising approach to solve the problem of imbalance between the spectrum scarcity and low utilization. The main idea contained in CR technology is that the secondary user (SU) can sense and exploit temporarily and local available licensed spectrum and adapt its radio parameter to opportunistically communicate over the spectrum of interest without harmfully interfering with the ongoing primary user (PU). In order to fully utilize the limited spectrum, the spectrum sharing strategy between the PU and SUs is an important issue. Two approaches to spectrum sharing have been addressed: spectrum overlay and spectrum underlay $[4,5]$. The spectrum underlay approach imposes restricted transmission power constraints on SUs so that they do not cause unacceptable interference to the PU. The advantage of this approach is that SUs can directly occupy the licensed spectrum without considering the behavior of the PU. However, SUs must carefully select their transmission power according to the severe power constraints. Also, they may suffer from the PU's interference. Differing from spectrum underlay, the spectrum overlay strategy does not necessarily impose transmission power restriction on SUs but rather than on when and where they can occupy the licensed spectrum. They can transmit only when the PU is inactive. The central issue of this approach is to identify the local and instantaneous unused spectrum with some detection probability.

Recently, there are several previous works addressing the M2M spectrum sharing problem by the help of the CR. For example, in [6], a spectrum sensing model based on Markov chain was proposed to predict the spectrum hole for $\mathrm{CR}$ in M2M network, and the theoretical analysis and simulation results were evaluated that a Markov model with two states or four states works well enough in wireless Internet of things (IoT) whereas a model with more states was not necessary. In [7], the authors proposed a machine-to-machine network management architecture based on cognitive network management technology and service-oriented architecture to provide effective and efficient network management of machine to machine network. In [8], the authors proposed a new M2M communications paradigm, namely cognitive M2M (CM2M) communication and presented CM2M communications architecture for the smart grid, for which also proposed an energy efficiency driven spectrum discovery scheme. In [9], the authors considered the heterogeneous characteristics of smart grid traffic including multimedia and developed $C R$ channel allocation and traffic scheduling schemes taking into consideration of channel switch and spectrum sensing errors and solve a system utility optimization problem for smart grid communication system.

In order to design the spectrum sharing strategies for M2M network, there are some more basic questions still remain unanswered. First, the M2M devices should take their locations into account when they adopt the spectrum sharing strategies. Specifically, if the M2M device is far from the PU, its transmission causes little interference to the PU if it carefully selects the transmitted power. In this case, the M2M device can occupy the spectrum by an underlay way; on the other hand, if the M2M device is near to the PU, in order to not cause the unacceptable interference to the PU, it is allowed to access the spectrum only when it detects that the PU is not active, i.e., the M2M device communicates over the spectrum by a overlay way. Therefore, considering the location of M2M devices, the overlay and underlay approaches can be employed simultaneously.

Motivated by the preceding discussion, in this paper, first, we give a M2M network communication scenario in which the spectrum is shared by a joint overlay and underlay approach and develop a connectivity establishment mechanism based on the cognitive radio technology. Then, we propose an optimal power allocation scheme in this scenario which holds the characteristics of achieving the maximum system utility and satisfying the QoS requirement of M2M devices and interference constraints of PU. Moreover, we propose a QoS sensitivity-based admission control algorithm to deal with the case that the optimal power allocation scheme is infeasible.

The rest of the paper is organized as follows. Section 2 gives a brief description of the system model and formulates the problems. Section 3 depicts the details of optimal power allocations algorithm. Our simulation results are given in Section 4. Section 5 concludes the paper.

\section{System model and problem description}

\subsection{Cognitive-based M2M network}

The cognitive-based M2M network we considered here is shown in Figure 1. The proposed M2M network is composed of a number of M2M devices, a licensed user (PU), and a dedicated cognitive M2M gateway, and the $\mathrm{PU}$ is not the M2M device.

First of all, in the proposed M2M scenario, the gateway is responsible for managing the whole M2M network, and the network related functionalities are implemented in the gateway, including connectivity establishment, access control, and QoS management. In particular, the M2M gateway provides the connectivity to the devices and the connection between the M2M network and other network, and the devices within the M2M network could directly communicate with the gateway based on the established connectivity to upload information.

Furthermore, we model a situation where $K$ M2M devices, each formed by a single transmitter-receiver pair, 


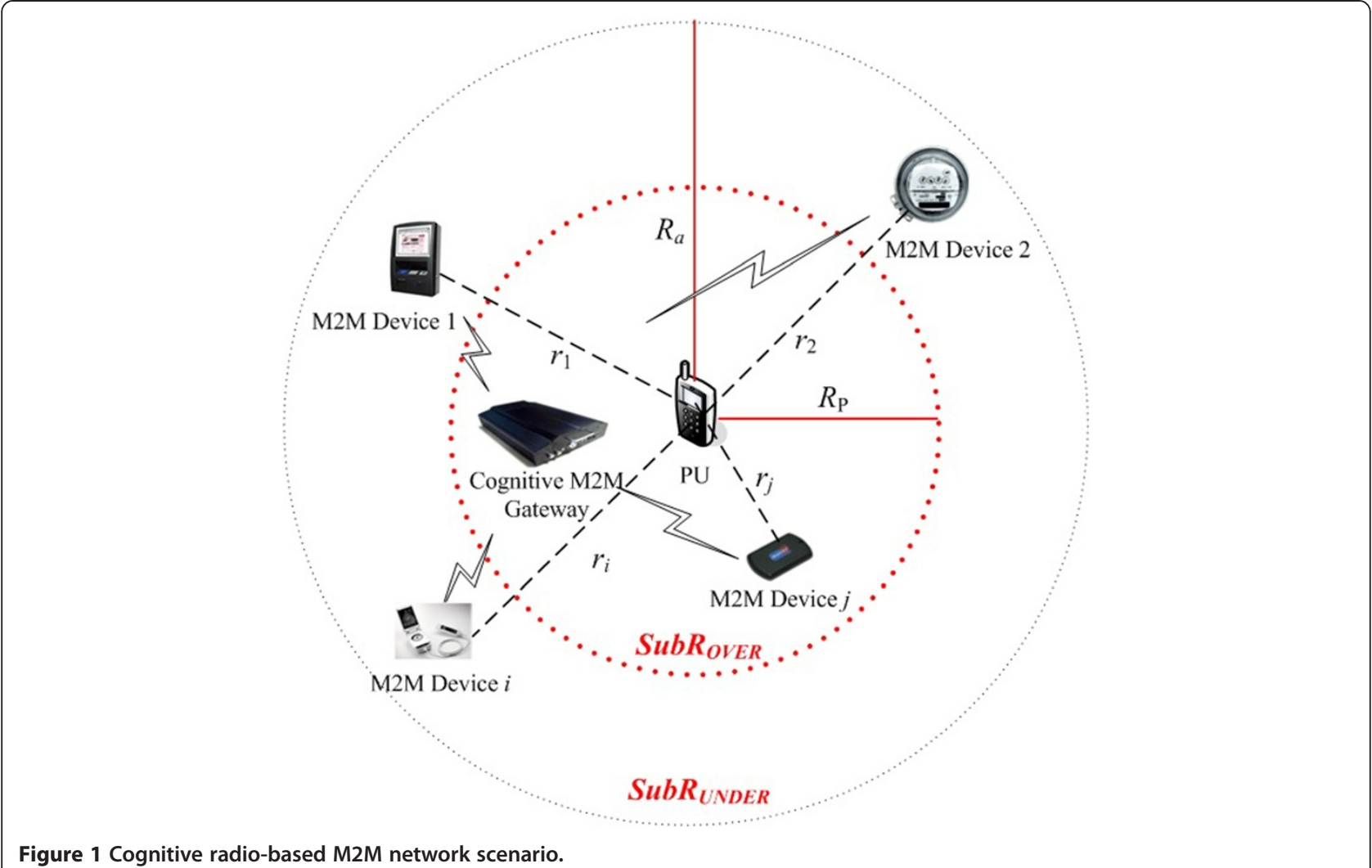

coexist and operate in a local circular region (the radius is denoted by $\mathrm{R}_{a}$ ) with a PU. The PU's receiver (PU Rx) with omnidirectional antenna is assumed to be the center of the circle. Both the M2M gateway and the devices satisfy uniform distribution in this region. We assume that the cognitive radio network is decentralized with slotted transmission structure. At each slot, the PU has a $\zeta_{\mathrm{P}}$ probability to access the spectrum, and each M2M device wants to opportunistically access the spectrum without causing the unacceptable interference to the PU Rx.

In order to facilitate the following discussion, we adopt the method in [10] to divide the circular region into two subregions, denoted by $S u b R_{\mathrm{OVER}}$ and $S u b R_{\mathrm{UNDER}}$, based on a carefully selected radius $R_{\mathrm{P}}\left(0<R_{\mathrm{P}} \leq R_{a}\right)$. SubR $R_{\mathrm{OVER}}$ is the region of the circle centered at PU Rx with radius $R_{\mathrm{P}} . S u b R_{\mathrm{UNDER}}$ denotes the region within a circle with radius $R_{a}$ but outside the circle with radius $R_{\mathrm{P}}$. In particular, the value of $R_{\mathrm{P}}$ mainly depends on the transmitted power of M2M device and the acceptable interference to the PU. Based on the definition of SubR OVER and $S u b R_{\text {UNDER }}$, we can classify M2M devices as two sets: the set $S_{\mathrm{UNDER}}=\left\{i \mid R_{\mathrm{P}}<R_{i} \leq R_{a}\right\}$ and the set $S_{\mathrm{OVER}}=$ $\left\{i \mid 0 \leq R_{i} \leq R_{\mathrm{P}}\right\}$, where $R_{i}$ denotes the distance between the transmitter of M2M Device ${ }_{i}$ (Device $_{i} \mathrm{Tx}$ ) and PU Rx. Since the M2M devices belonging to $S_{\text {OVER }}$ are near to the PU Rx, they can only adopt overlay approach to transmit information in order to not cause unacceptable interference to PU Rx and guarantee themselves QoS requirements (in terms of minimum required signal-tointerference-plus-noise ratio, SINR). For the devices in $S_{\text {UNDER }}$, since they are relative far from the PU Rx, they can use the spectrum in an underlay way without considering the PU's usage. But these devices must carefully select their transmission power in order to make sure that the sum interference they cause is acceptable to the PU RX.

In order to simplify the following discussion, we have adopted deterministic signal propagation model without considering the fading and shadowing. Note that this signal propagation model can be applied to general scenarios.

In the proposed architecture, the communication within the proposed M2M network typically consists of four phases: connectivity establishment, data collection, data transmission, and data processing. The connectivity establishment phase refers to the procedure used to exploit the temporarily and local available licensed spectrum and then establish the communication link between the device and the gateway based on the available spectrum. The data collection phase is the process executed by the devices to obtain the physical data. The data transmission phase includes the communication between the M2M gateway and devices and the M2M gateway and an external entity, respectively. The data 
processing phase is the process of dealing with and analyzing the data.

It is noteworthy that the connectivity establishment between the devices and the M2M gateway is the fundamental process for the M2M network communication. In this section, we adopt a cognitive-based connectivity establishment scheme, and more details are described in [11]. In the following, we will elaborate on the connectivity establishment mechanism and the performance analysis. Moreover, the main difference between the connectivity establishment mechanism based on cognitive radio and the traditional one is that the $\mathrm{M} 2 \mathrm{M}$ devices can dynamically use the white spectrum based on the spectrum environment and the demands which can provide more flexibility.

Moreover, the key feature of the cognitive radio-based M2M network proposed in this paper is the capability of recognizing the communication environment and adaptively modifying the communication parameters, i.e., the M2M devices could sense the licensed spectrum based on the gateway kick-off information and then occupy the white spectrum holes. In order to improve the spectrum sensing performance and decrease the false detection probability, we adopt the distributed spectrum sensing scheme, that is, each M2M device self-organized senses the target spectrum and then returns the sensing results to the cognitive gateway. The gateway collects the available spectrum information and help to establish the connectivity. Based on the aforementioned discussion, the cognitive radio-based connectivity establishment mechanism in M2M network can be depicted in Figure 2, which mainly contains seven main steps:

(1) Kick-off: the M2M gateway informs the M2M devices through a predefined channel to start the spectrum sensing procedure.

(2) Self-organization spectrum sensing: each M2M device independently implements the spectrum sensing to find the idle spectrum.
(3) Collection of the available spectrum: the M2M gateway collects the available spectrum from the devices and selects an idle sub-spectrum to establish the connectivity between the related device and the gateway.

(4) Spectrum selection confirmation: the gateway sends a notice to the device to confirm the selected idle sub-spectrum.

(5) Connectivity establishment: both of the gateway and the device configure their RF with the selected spectrum and establish the connectivity.

(6) Information transmission: the device and the gateway exchange information using the established connectivity. Furthermore, in the most cases of M2M communication scenario, information transmission will occupy the uplink channel, which means the M2M device report its collected data to the gateway and behind server.

(7) Release the connectivity: both the device and the gateway disconnect the link.

To avoid the situation where the PU reuse the licensed spectrum occupying by the M2M network, we assume that the M2M device continues to periodically sense the target spectrum. More specifically, over each time slot and in a target spectrum, as illustrated in Figure 3, the frame structure consists of a spectrum sensing slot and a data transmission slot. For the $i$ th target spectrum, suppose that the frame duration and the sensing duration at frame tag $t$ is denoted by $T$ and $\tau_{i, t}$, respectively, and the date transmission duration is $T-\tau_{i, t}$.

Furthermore, we suppose that the PU maintains the same spectral-temporal usage characteristics in one frame. In practice, this assumption is reasonable since IEEE 802.22 has developed an air interface for opportunistic secondary access to the TV spectrum in which the primary signals change slowly, and the frame duration should be designed to be smaller than the average duration of the PU's transmission.

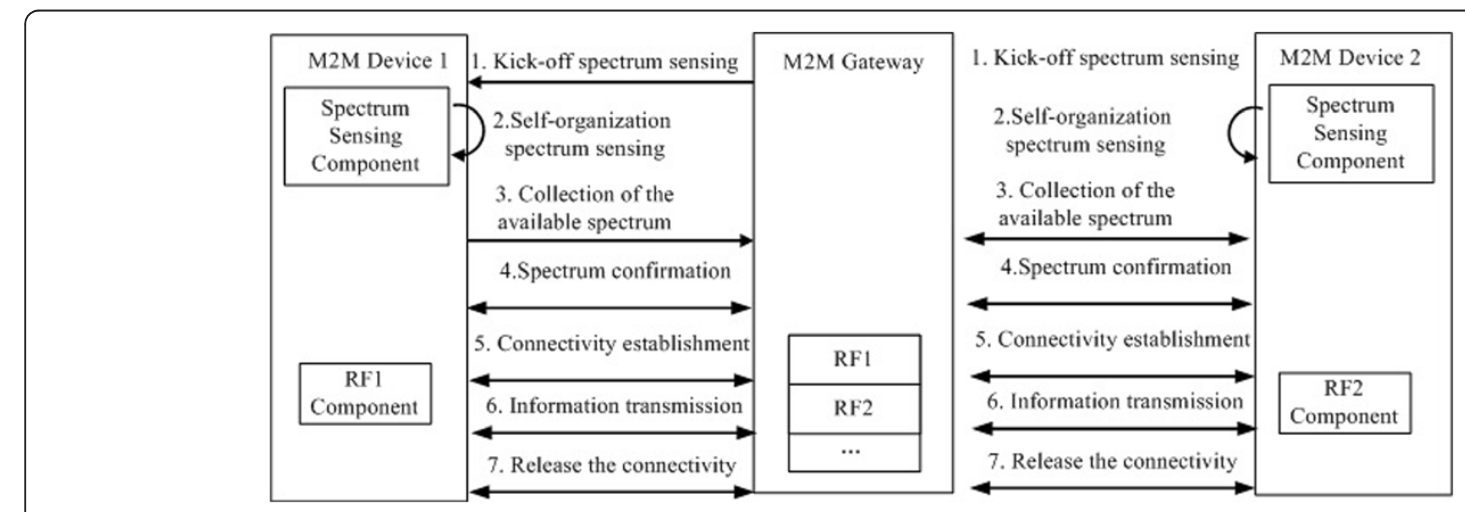

Figure 2 Connectivity establishment mechanism. 


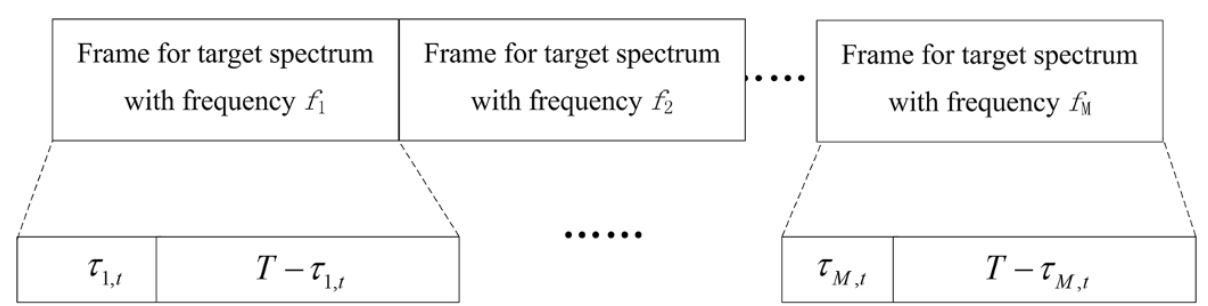

Figure 3 Frame structure for interleaved sensing and utilization over the target spectrum of interest. $\tau_{i, t,}$ sensing slot duration; $T-\tau_{i, t,}$ data transmission slot duration.

It also notes that the M2M gateway will coordinate the spectrum selection conflict in selecting the idle subspectrum.

\subsection{Spectrum sensing scheme over the target channel}

Whether for the detection of the spectrum holes, the spectrum sensing involves deciding whether the primary signal is present or not from the observed signals. We suppose that the received signal at the $i$ th $\mathrm{M} 2 \mathrm{M}$ device is sampled at sampling frequency $f_{s}$ over the target channel. The primary signal detection problem can be modeled as the following binary hypotheses:

$$
x_{i}(n)=\left\{\begin{array}{cc}
\eta_{i}(n), & \mathcal{H}_{0, i} \\
h_{i} s_{i}(n)+\eta_{i}(n), & \mathcal{H}_{1, i}
\end{array}\right.
$$

where $x_{i}(n)$ is the $n$th received sample, $s_{i}(n)$ is the primary transmitted signal, and each sample is assumed to be independent and identically distributed (i.i.d) with zero mean and variance $\sigma_{s}^{2}$, i.e., $s_{i}(n)^{\sim} \mathcal{C N}\left(0, \sigma_{s}^{2}\right), \eta_{i}(n)$ is the noise and assumed to be i.i.d circularly symmetric complex Gaussian with zero mean and variance $\sigma_{\eta}^{2}, \eta_{i}(n)$ $\sim \mathcal{C N}\left(0, \sigma_{\eta}^{2}\right)$. Moreover, $h_{i}$ is the target channel gain between the primary transmitter and the $i$ th $\mathrm{M} 2 \mathrm{M}$ device receiver with the variance $\mathbf{E}\left(\left|h_{i}\right|^{2}\right)=\sigma_{h, i}^{2}, \mathcal{H}_{0, i}$ and $\mathcal{H}_{1, i}$ denote the hypotheses corresponding to the absence and presence of the PU, respectively. Suppose $s_{i}(n), \eta_{i}(n)$, and $h_{i}(n)$ are independent of each other and the average received signal-to-noise ratio (SNR) can be calculated by

$$
\gamma_{i}=\frac{\sigma_{s}^{2} \sigma_{h, i}^{2}}{\sigma_{\eta}^{2}} .
$$

Let $N_{t}$ denote the number of samples over the target channel; without loss of the generality, we assume that $N_{i}=\tau_{i} f_{s}$. The test statistics is thus obtained as the observed energy summation within $N_{t}$ consecutive components, i.e.,

$$
X_{i}= \begin{cases}\frac{1}{N_{i}} \sum_{j=1}^{N_{i}}\left|\eta_{i}(j)\right|^{2}, & \mathcal{H}_{0, i} \\ \frac{1}{N_{i}} \sum_{j=1}^{N_{i}}\left|h_{i} s_{i}(j)+\eta_{i}(j)\right|^{2} . & \mathcal{H}_{1, i}\end{cases}
$$

Then under hypothesis $\mathcal{H}_{0, i}$, the test statistic $X_{i}$ follows a central chi-square distribution with $2 N_{i}$ degrees of freedom and a noncentral chi-square distribution with $2 N_{i}$ degrees of freedom and a noncentrality parameter $2 \gamma_{i}$ for $\mathcal{H}_{1, i}$. i.e.,

$$
f_{X_{i}}\left(X_{i}\right)= \begin{cases}\chi_{2 N_{i}}^{2} & \mathcal{H}_{0, i} \\ \chi_{2 N_{i}}^{2}\left(2 \gamma_{i}\right), & \mathcal{H}_{1, i}\end{cases}
$$

where $f_{X_{i}}\left(X_{i}\right)$ denotes the probability density function (PDF) of $X_{i}$.

The performance of spectrum sensing algorithm in M2M network can be characterized by two parameters: probability of detection $P_{d}$ and probability of false alarm $P_{f} . P_{d}$ is the probability of detecting the presence of the $\mathrm{PU}$, while $P_{f}$ is the probability that the test incorrectly decides that the considered frequency is occupied by the PU when it actually is not. The probabilities of detection and false alarm have unique implications for both the PU and M2M devices. More specifically, from the PU's point of view, a large detection probability is desired in order to sufficiently protect the PU from the interference caused by the M2M device. From the M2M device's perspective, $P_{f}$ should be kept as small as possible for preventing underutilization of transmission opportunities. Therefore, the design of a detection algorithm leads to a tradeoff between the probability of detection and the probability of false alarm.

In order to facilitate the following discussion, we assume that the PU's signal is a complex-valued PSK signal. Based on the PDF of the test statistic, using central limit theorem, for large $N_{i}$, the energy detector's probabilities of detection and false alarm for the $i$ th target channel can be approximated as [12]

$$
P_{d, i}\left(\tau_{i}, \varepsilon_{i}, \gamma_{i}\right)=Q\left(\left(\frac{\varepsilon_{i}}{\sigma_{\eta}^{2}}-\gamma_{i}\left|h_{i}\right|^{2}-1\right)\left(\frac{\tau_{i} f_{s}}{2 \gamma_{i}\left|h_{i}\right|^{2}+1}\right)^{\frac{1}{2}}\right),
$$

$$
P_{f, i}\left(\tau_{i}, \varepsilon_{i}\right)=Q\left(\left(\frac{\varepsilon_{i}}{\sigma_{\eta}^{2}}-1\right)\left(\tau_{i} f_{s}\right)^{\frac{1}{2}}\right)
$$


where $\varepsilon_{i}$ denotes the detection thresholds, $Q($.$) is the$ complementary distribution function of the normalized Gaussian given by

$$
Q(x)=\frac{1}{\sqrt{2 \pi}} \int_{x}^{\infty} \exp \left(\frac{-u^{2}}{2}\right) d u .
$$

Furthermore, the probability of missed detection for the $i$ th $\mathrm{M} 2 \mathrm{M}$ device is defined as

$$
P_{m, i}\left(\tau_{i}, \varepsilon_{i}, \gamma_{i}\right)=1-P_{d, i}\left(\tau_{i}, \varepsilon_{i}, \gamma_{i}\right) .
$$

To evaluate the performance of the energy detection algorithm, the main metric is either minimizing the miss probability for a target false alarm probability or minimizing the false alarm probability for a target miss probability. Thus, for a targeted detection probability $P_{d, i}$, the probability of the false alarm is given by plugging (5) into (6),

$$
P_{f, i}\left(\tau_{i}\right)=Q\left(Q^{-1}\left(p_{d, i}^{T}\right) \sqrt{2 \gamma_{i}\left|h_{i}\right|^{2}+1}+\gamma_{i}\left|h_{i}\right|^{2} \sqrt{\tau_{i} f_{s}}\right)
$$

While, for a targeted false alarm probability $p_{f, i}^{T}$, the probability of the detection is given by substituting (6) into (5),

$$
P_{d, i}\left(\tau_{i}\right)=Q\left(\frac{Q^{-1}\left(p_{f, i}^{T}\right)-\gamma_{i}\left|h_{i}\right|^{2} \sqrt{\tau_{i} f_{s}}}{\sqrt{2 \gamma_{i}\left|h_{i}\right|^{2}+1}}\right) .
$$

\subsection{M2M network power allocation algorithm formulation} In this section, we follow the method in [10] to formulate the power allocation algorithm. In the joint spectrum overlay and underlay M2M network, there exist some basic problems that must be carefully investigated. The most important two are power allocations and interference managements of M2M devices. An ideal power allocation scheme should maximize the system utility of M2M network while causing acceptable interference to the PU. In this paper, we assume that both the M2M devices in $S_{\mathrm{N}}$ and $S_{\mathrm{F}}$ adopt a spread spectrum signaling format in which the transmitted power is evenly spread across the entire available spectrum.

For any devices $\alpha$ in $S_{\mathrm{UNDER}}$, its SINR can be expressed as

$$
\mu_{\alpha}=\frac{P_{\mathrm{S} \alpha} G_{\alpha \alpha}}{n_{0}+\sum_{i \neq \alpha} P_{\mathrm{S} i} G_{i \alpha}+P_{\mathrm{P}} G_{\mathrm{P} \alpha}}
$$

where $n_{0}$ is the background noise power that is assumed to be the same for all users, $G_{\alpha \alpha}$ is the channel direct gain for M2M device $\alpha^{\prime}$ transmitter to its receiver, $G_{i \alpha}$ $\left(G_{\mathrm{P} \alpha}\right)$ is the channel cross gain from M2M Device ${ }_{i}$ $(\mathrm{PU})$ 's transmitter to $\alpha$ 's receiver, $P_{\mathrm{S} i}\left(P_{\mathrm{P}}\right)$ is the transmitted power of M2M Device D $\left._{i} \mathrm{PU}\right)$. Note that in (11), we actually consider the worst case of PU interference by treating the PU as all being active, i.e., the access probability of PU is equal to 1 .

For any devices $\beta$ in $S_{\text {OVER }}$, its SINR can be represented by

$$
\mu_{\beta}=\frac{P_{\mathrm{S} \beta} G_{\beta \beta}}{n_{0}+\sum_{i \neq \beta} P_{S i} G_{i \beta}} .
$$

Since the PU is not active when $\beta$ accesses the spectrum, $\beta$ does not suffer from the PU interference. In order to guarantee the M2M device's QoS, the power allocations scheme should satisfy the following SINR constraints

$$
\mu_{i} \geq \beta_{i} \quad i=1,2, \ldots K
$$

Here, $\beta_{i}$ is the required SINR corresponding to the desired value of bit error rate. Let $I_{\mathrm{P}}$ denote the interference threshold tolerable at PU Rx; the power allocations scheme must satisfy the interference constraint

$$
\sum_{\alpha \in S_{\mathrm{UNDER}}} P_{\mathrm{S} \alpha} G_{\alpha \mathrm{P}} \leq I_{\mathrm{P}}
$$

where $G_{\alpha \mathrm{P}}$ is the channel cross gain from $\alpha$ 's transmitter to PU Rx. Note that devices in $S_{\text {OVER }}$ are forbidden to access the spectrum when PU transmits information, so they contribute no interference to the PU. Recall the distribution assumption of devices, the number of devices in $S_{\mathrm{UNDER}}$ (denoted by $n_{\mathrm{F}}$ ) can be calculated by

$$
n_{\mathrm{F}}=K \frac{R_{a}^{2}-R_{\mathrm{P}}^{2}}{R_{a}^{2}}
$$

where $\lfloor n\rfloor$ denotes the integer part of $n$. It is easy to find that $n_{\mathrm{F}}$ is a monotone decreasing function in $R_{\mathrm{P}}$. When $R_{\mathrm{P}}$ is small, the number of devices in $S_{\mathrm{F}}$ is corresponding large. Thus, the performance of PU will be declined because of the unacceptable interference caused by the devices in $S_{\mathrm{F}}$. In contrast, when $R_{\mathrm{P}}$ is large, the number of devices in $S_{\mathrm{N}}$ is correspondingly large. If the $\mathrm{PU}$ has high access probability, then, the system utility of devices will be decreased because there are only a few devices that can use the spectrum by an underlay way. So $R_{\mathrm{P}}$ must be carefully investigated when designing the power allocation scheme.

In order to evaluate the performance of the M2M Device $_{i}$, we adopt the following utility function as the metric

$$
\mathrm{U}_{\mathrm{S} j}=\left\{\begin{array}{ll}
\ln \left(\mu_{j}\right) & \text { if } j \in S_{\mathrm{UNDER}} \\
\left(1-P_{d, j}\left(\tau_{j}\right)\right) \ln \left(\mu_{j}\right) & \text { if } j \in S_{\mathrm{OVER}}
\end{array} .\right.
$$


For each time slot, since the M2M devices belonging to $S_{\text {OVER }}$ have $\left(1-P_{d, j}\left(\tau_{j}\right)\right)$ probability to access the spectrum, we add access probability multiple to the utility function of devices in $S_{\text {OVER }}$.

\section{Optimal power allocation scheme}

One of the most important goals we want to achieve is the optimal power allocation for different M2M devices. Specifically, the power allocation scheme should coordinate the device's transmitted power to achieve the maximum system utility. In this section, we assume that the devices in $S_{\mathrm{N}}$ can perfect sense the PU's activity, i.e., they can exactly sense the PU's behavior in a particular slot.

3.1 The power allocation scheme with utility function Based on the aforementioned discussion, the optimal power allocation problem can be expressed as follows:

$$
\begin{array}{ll}
\max _{P_{\mathrm{S} 1}, \ldots P_{\mathrm{SK}}} & \left(\sum_{\alpha \in S_{\mathrm{UNDER}}, \alpha=1}^{n_{\mathrm{F}}} U_{\mathrm{S} \alpha}+\sum_{\beta \in S_{\mathrm{OVER}}, \beta=1}^{K-n_{\mathrm{F}}} \mathrm{U}_{\mathrm{S} \beta}\right), \\
\text { s.t. } \quad & 0 \leq P_{\mathrm{S} j} \leq P_{\mathrm{S} j}^{\mathrm{Max}} j=1,2, \ldots K, \\
& \sum_{\alpha \in S_{\mathrm{UNDER}}} \geq \beta_{i} i=1,2, \ldots K,
\end{array}
$$

where $P_{\mathrm{S} j}^{\mathrm{Max}}$ is the maximum transmitted power of M2M Device $_{i}$. Note that the objective function $\sum_{\alpha \in S_{\mathrm{UNDER}, \alpha=1}}^{n_{\mathrm{F}}} U_{\mathrm{S} \alpha}+$ $\sum_{\beta \in S_{\mathrm{OVER}}, \beta=1}^{K-n_{\mathrm{F}}} \mathrm{U}_{\mathrm{S} \beta}$ is equivalent to $\ln \left(\left(\prod_{j \in \mathrm{S}_{\mathrm{UNDER}}, j=1}^{\prod_{\mathrm{F}}} \mu_{j}\right)\right.$ $\left.\left(\left(\prod_{i \in \mathrm{S}_{\text {OVER }}, i=1}^{K-n_{\mathrm{F}}} \mu_{i}\right)^{\left(1-P_{d, i}\left(\tau_{i}\right)\right)}\right)\right)$ which provides the well-known proportional fair among devices [13]. Moreover, if $P_{d, i}$ $\left(\tau_{i}\right)=0$, i.e., the PU is inactive in the slot, the objective function can be reduced to $\prod_{i=1}^{K} \mu_{i}$; otherwise, if $P_{d, i}$ $\left(\tau_{i}\right)=1$, i.e., the PU occupies the spectrum all the time, the objective function can be reduced to $\prod_{i=1}^{\mathrm{N}_{\mathrm{F}}} \mu_{i}$. The above two extreme cases are easier solved. Thus, in this paper, we mainly consider the case $0<P_{d, i}\left(\tau_{i}\right)<1$. It is easy to find that (17) is a constraint optimization problem, it can be solved by the method of generalized geometric programming [14].

\subsection{Power allocation scheme with optimal achievable throughput}

In this subsection, we propose a power allocation scheme to achieve the optimal achievable throughput.
The throughput of any M2M device $\alpha$ in $S_{\text {UNDER }}$ can be expressed as

$$
T_{\alpha}=W \log _{2}\left(1+\frac{P_{\mathrm{S} \alpha} G_{\alpha \alpha}}{n_{0}+\sum_{i \neq \alpha} P_{\mathrm{S} i} G_{i \alpha}+P_{\mathrm{P}} G_{\mathrm{P} \alpha}}\right),
$$

where $W$ is the spectrum bandwidth.

The throughput of any device $\beta$ in $S_{\text {OVER }}$ can be represented by

$$
T_{\beta}=\left(1-P_{d, \beta}\left(\tau_{\beta}\right)\right) W \log _{2}\left(1+\frac{P_{S \beta} G_{\beta \beta}}{n_{0}+\sum_{i \neq \beta} P_{S i} G_{i \beta}}\right)
$$

Since the central issue that we want to investigate is the throughput of each device, it is important whether or not a power allocation scheme can yield a requested throughput vector $\mathbf{T}=\left[T_{1}, T_{2}, \ldots T_{K}\right]$. A throughput vector is called an achievable throughput vector of a power allocation scheme if the scheme can yield the throughput $T_{i}$ of M2M Device ${ }_{i}$, and the achievable throughput region is defined by the set of all achievable throughput vectors

$$
\begin{aligned}
& T=\left\{\mathbf{T}: T_{\alpha} \forall \alpha \in S_{\mathrm{UNDER}}, T_{\beta} \forall \beta \in S_{\mathrm{OVER}}, \text { and } P_{\mathrm{S} j} \leq P_{S j}^{\mathrm{Max}},\right. \\
& \left.j=1,2, \ldots, K, \sum_{\alpha \in S_{\mathrm{UNDER}}} P_{\mathrm{S} \alpha} G_{\alpha \mathrm{P}} \leq I_{\mathrm{P}}, \text { and } P_{\mathrm{S} \alpha}, P_{\mathrm{S} \alpha} \text { satisfies }(13)\right\}
\end{aligned}
$$

Let $T^{*}$ be the set of Pareto optimal points of $T[14]$

$$
\begin{array}{r}
T^{*}=\left\{\left(T_{1}, T_{2}, \ldots T_{K}\right) \in T: T_{i} \geq \widetilde{T}_{i} \forall\left(T_{1}, \ldots T_{i-1}, \widetilde{T}_{i},\right.\right. \\
\left.\left.T_{i+1}, T_{K}\right) \in T, \text { for } i=1,2, \ldots K\right\} .
\end{array}
$$

Therefore, a throughput is Pareto optimal (or efficient) if it is not possible to increase the throughput of any device without decreasing the throughput of some other devices.

Note that the above optimization problem is a nonlinear optimization one; it can be solved by the method of Karush-Kuhn-Tucker (KKT).

\section{Simulation results}

In this section, we present the simulation results. All simulations consist of the following setup: $n_{0}=0.01, K$ varies from 2 to $80, R_{\mathrm{P}}$ varies from 20 to $100, G_{\mathrm{SiS} i}=2$, $P_{\mathrm{S} i}^{\mathrm{Max}}=2, \beta_{i}=0.1, I_{\mathrm{P}}=4, R_{a}=100, G_{\mathrm{SiSj}}=0.1, i \neq j, G_{\mathrm{PS} i}=$ $G_{\text {SiP }}=0.02, W=1$. We also assume the M2M devices satisfy uniform distribution with density $\lambda$, and here, we set $\lambda=1$. 


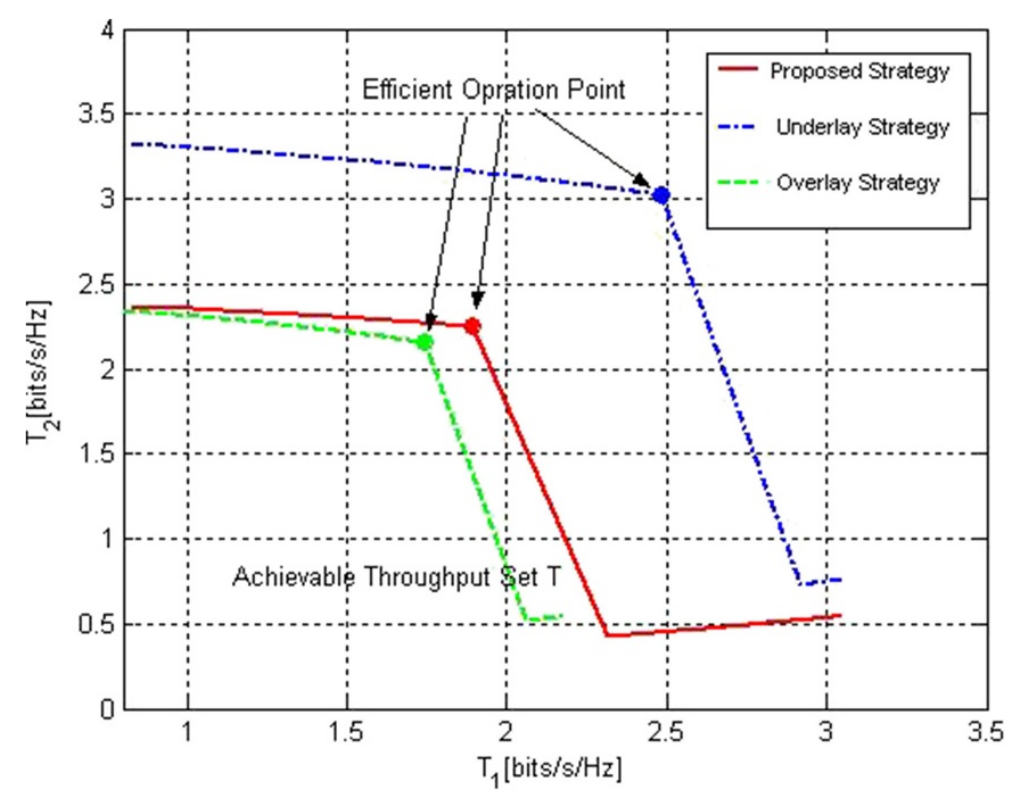

Figure 4 Achievable throughput set $T$ and Pareto optimal points set $T^{*}$ for different strategy.

In Figure 4, without loss of generality, we consider two M2M devices case where user $S_{1}$ and $S_{2}$ belong to $S_{\text {UNDER }}$ and $S_{\text {OVER }}$, respectively. It is easy to extend the results to the multiple M2M devices scenarios. We show the achievable throughput set $T$ and the Pareto optimal points set $T^{*}$ for different strategies. It can be seen that the achievable throughput set $T$ of our proposed strategy is more efficient than the overlay strategy. Although the throughput of our strategy is smaller than the underlay strategy, it causes less interference to the PU comparing to the underlay strategy; thus, the proposed strategy can ensure the better QoS of the PU.
Figure 5 depicts the M2M network utility in terms of the spectrum sensing time $\tau$ for different numbers of M2M devices. Here, we set the number of the target subchannels sensed by the M2M devices $J=10$. We can see that the network utility is increasing with $M$ (the number of M2M devices). It is noted that the utility is saturated when $M$ is larger. Thus, for a targeted $M_{\max }$, it is not an optimal solution to more M2M devices.

\section{Conclusions}

In this paper, we have considered the power allocation problem in joint spectrum overlay and underlay $\mathrm{M} 2 \mathrm{M}$

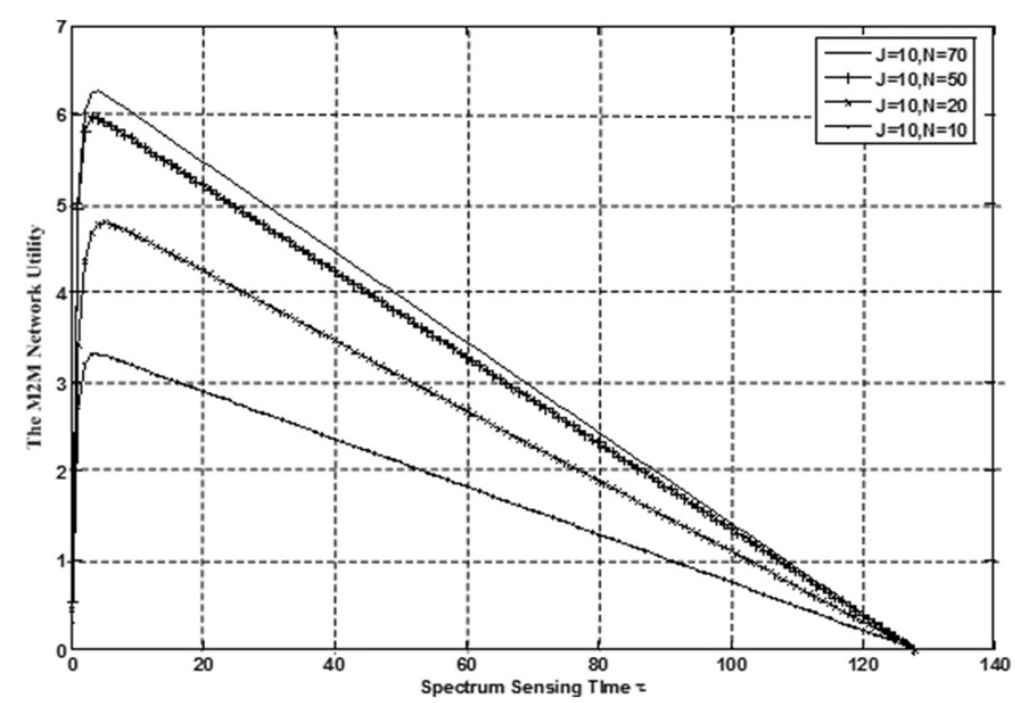

Figure 5 The M2M network utility vs. the spectrum sensing time $\tau$ for different numbers of M2M devices $N$. 
networks. We develop a connectivity establishment mechanism in M2M network based on the cognitive radio technology and proposed an optimal power allocation scheme aiming at maximizing the system utility and satisfied the QoS of M2M devices and interference of PU at the same time.

\section{Competing interests}

The authors declare that they have no competing interests.

\section{Acknowledgement}

This work was supported by NSFC $(61379016,61271180)$, Important National Science and Technology Specific Projects (2013ZX03001020-003), Doctoral Program of Higher Education (20130005110016), and BUPT Youth Research and Innovation Plan (2014RC0103).

\section{Author details}

${ }^{1}$ State Key Lab of Networking and Switching Technology, Beijing University of Posts and Telecommunications, Beijing 100876, China. ${ }^{2}$ China Transport Telecommunication and Information Center, Beijing 100876, China. ${ }^{3}$ The State Radio Monitoring Center, Beijing 100876, China.

Received: 3 April 2014 Accepted: 5 May 2014

Published: 21 May 2014

\section{References}

1. C Inhyok, Y Shah, AU Schmidt, A Leicher, MV Meyerstein, Trust in M2M communication. IEEE Vehic. Tech. Mag. 4(3), 69-75 (2009)

2. $Y$ Zhang, $R$ Yu, S Xie, W Yao, Y Xiao, M Guizani, Home M2M networks: architectures, standards, and QoS improvement. IEEE Commun. Mag. 49(4), 44-52 (2011)

3. J Mitola III, G Maguire Jr, Cognitive radio: making software radios more personal. Pers. Commun. IEEE [see also IEEE Wireless Commun] 6(4), 13-18 (1999)

4. S Haykin, Cognitive radio: brain empowered wireless communications. IEEE J. Selected Area Commun. 23(2), 201-220 (2005)

5. Z Z Qing, BM Sadler, A survey of dynamic spectrum access: signal processing, networking, and regulatory policy. IEEE Signal Process. Mag. 55(5), 2294-2309 (2007)

6. W Zhigang, L Jie, Spectrum sensing model for wireless internet of things. China Commun. 8(1), 8-13 (2011)

7. Q Yifang, S Qiang, L Tao, Z Zhijun, T Hui, C Song, F Zhiyong, Cognitive network management in internet of things. China Commun. 8(1), 1-7 (2011)

8. Y Zhang, R Yu, M Nekovee, Y Liu, S Xie, S Gjessing, Cognitive machine-tomachine communications: visions and potentials for the smart grid. IEEE Netw. 26(3), 6-13 (2012)

9. J Huang, H Wang, Y Qian, C Wang, Priority-based traffic scheduling and utility optimization for cognitive radio communication infrastructure-based smart grid. IEEE Trans. Smart Grid. 4(1), 78-86 (2013)

10. Y Haipeng, Z Zhou, L Zhang, H Liu, L Tang, KS Kwak, An efficient power allocation scheme in joint spectrum overlay and underlay cognitive radio networks (IEEE 9th International Symposium on Communications and Information Technology (ISCIT2009), Icheon, 2009), pp. 102-105

11. H Yao, W Wang, Y Liu, J Zhang, Spectrum sharing and power allocation analysis in machine to machine network. J. Conv. Inf. Technol. 7(19), 242-250 (2012)

12. H Seo, BG Lee, Proportional-fair power allocation with CDF-based scheduling for fair and efficient multiuser OFDM systems. IEEE Trans. Wireless Commun. 5(5), 978-983 (2006)

13. R Tandra, A Sahai, SNR walls for signal detection. IEEE J. Select. Topics Sig. Proc. 2, 4-17 (2008)

14. S Boyd, L Vandenberghe, Convex Optimization (Cambridge University Press, Cambridge, 2004)

doi:10.1186/1687-1499-2014-82

Cite this article as: Yao et al:: Optimal power allocation in cognitive radio based machine-to-machine network. EURASIP Journal on Wireless Communications and Networking 2014 2014:82.

\section{Submit your manuscript to a SpringerOpen ${ }^{\circ}$ journal and benefit from:}

- Convenient online submission

- Rigorous peer review

- Immediate publication on acceptance

- Open access: articles freely available online

- High visibility within the field

- Retaining the copyright to your article

Submit your next manuscript at springeropen.com 